\title{
Enalapril retards glomerular basement membrane thickening and albuminuria in the diabetic rat
}

\author{
M.E.Cooper ${ }^{1}$, T.J.Allen ${ }^{1}$, P.A. Macmillan ${ }^{1}$, B.E.Clarke ${ }^{2}$, G. Jerums ${ }^{1}$ and A.E. Doyle ${ }^{1}$ \\ ${ }^{1}$ Endocrine Unit, Department of Medicine and ${ }^{2}$ Department of Anatomical Pathology, Austin Hospital, Heidelberg, Victoria, Australia
}

\begin{abstract}
Summary. This study has evaluated the effects of the angiotensin converting enzyme inhibitor Enalapril on glomerular ultrastructure and albuminuria in normotensive and hypertensive diabetic rats. Streptozotocin-diabetes was induced in Wistar Kyoto and spontaneously hypertensive rats. Enalapril was administered in drinking water in diabetic normotensive, control hypertensive and diabetic hypertensive rats. Enalapril therapy prevented an increase in glomerular basement membrane thickness in diabetic normotensive, control hypertensive and diabetic hypertensive rats without any significant ef-
\end{abstract}

fect on fractional mesangial volume. Enalapril decreased albuminuria in diabetic normotensive, control hypertensive and diabetic hypertensive rats. Thus, enalapril retards the development of glomerular basement membrane thickening and albuminuria in the rat, in the presence or absence of hypertension.

Key words: Diabetes, hypertension, nephropathy, enalapril, albuminuria
Angiotensin converting enzyme (ACE) inhibitors have been shown to decrease albuminuria in hypertensive Type 1 (insulin-dependent) diabetic patients with established nephropathy [1] as well as in normotensive Type 1 diabetic patients with microalbuminuria [2]. In experimental diabetes, the ACE inhibitor, enalapril, has been shown to prevent the development of albuminuria and glomerulosclerosis in the streptozotocin (STZ) diabetic Munich-Wistar rats [3].

We have developed a model combining genetic hypertension with diabetes by inducing STZ diabetes in the spontaneously hypertensive rat (SHR) [4]. The diabetic SHR model develops albuminuria at a more rapid rate and has increased glomerular basement membrane thickness when compared to normotensive Wistar Kyoto (WKY) diabetic rats [4]. The diabetic WKY develops, when compared to the non-diabetic WKY, a five-fold increase in albuminuria, increased fractional mesangial volume and thicker glomerular basement membranes after 32 weeks of diabetes [4]. In preliminary studies, we have reported that the ACE inhibitor, enalapril, retards the development of albuminuria in both hypertensive and normotensive diabetic rats [5]. In the present study, we have followed animals for 32 weeks after induction of diabetes and have evaluated the effects of ACE inhibition on glomerular ultrastructure.

\section{Materials and methods}

Male SHR (Okamoto) and normotensive WKY from our inbred stock weighing between 200 and $250 \mathrm{~g}$, aged between 8 and 9 weeks, were injected intravenously with STZ according to a previously reported protocol [4]. In the normotensive WKY strain, all animals were injected with STZ and then randomised to receive either no treatment, or enalapril (Merck, Sharp \& Dohme, Rahway, NJ, USA) $35 \mathrm{mg} / \mathrm{l}$ in drinking water. In the SHR strain, two thirds of the animals were injected with STZ and the remaining third served as non-diabetic, hypertensive controls. Both the diabetic and control SHR were then randomised to receive either no treatment or enalapril. Since control animals drink less than diabetic animals, the dose of enalapril was doubled to $70 \mathrm{mg} / 1$ in drinking water for control SHR whereas diabetic SHR received $35 \mathrm{mg} / 1$.

All rats were caged in groups of four and fed a normal diet (GR $2+$ rat cubes, Clarke King and Co., Melbourne, Australia) containing $20 \%$ protein. At 4 -weekly intervals over the 32 -week study period, animals were weighed and blood-pressure was measured by indirect tailcuff plethysmography in unanaesthetised, preheated rats. Serum glucose was measured by a glucose oxidase technique and urinary albumin excretion was measured by a coated tube radioimmunoassay as previously described [4]. Renal histology was assessed by quantitative histomorphometric methods [4] at the end of the 32-week study period after in vivo fixation of tissues by intra-arterial perfusion with $2.5 \%$ glutaraldehyde. Glomerular basement membrane (GBM) thickness was measured by the orthogonal intercept method [4], glomerular volume was measured by light microscopy using a point counting method in which a minimum of 50 glomeruli were assessed per rat and mesangial expansion was assessed by calculation of the fractional mesangial volume as previously described [4]. In brief, mesangium was identified in electron micrographs by an independent observer (B.C.) and using a point counting method the percentage of glomeru- 
Table 1. Weight, glycaemic control, albuminuria and glomerular ultrastructure

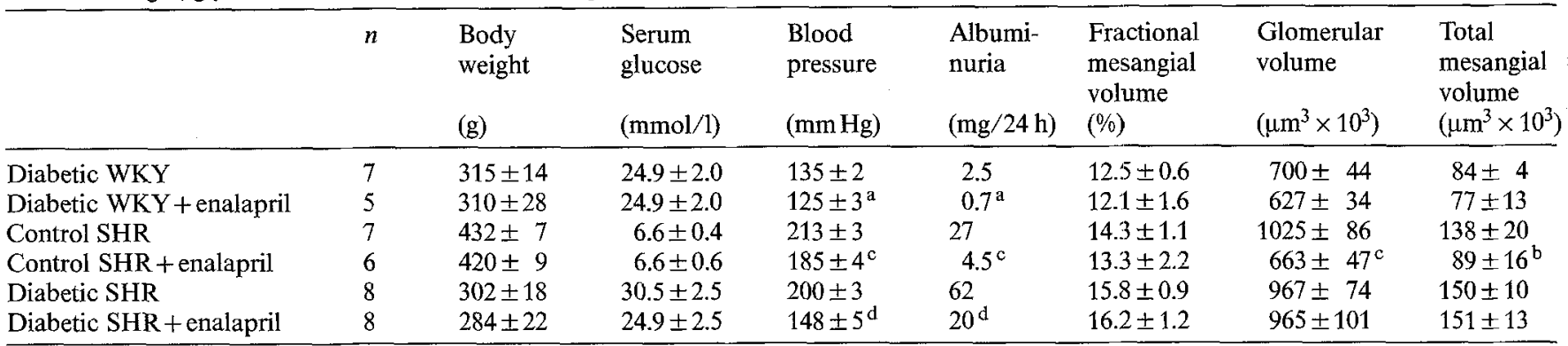

$\mathrm{WKY}=$ Wistar Kyoto rat; $\mathrm{SHR}=$ Spontaneously hypertensive rat

${ }^{\mathrm{a}}-p<0.05$ vs diabetic WKY ${ }^{\mathrm{b}}-p<0.05,{ }^{\mathrm{c}}-p<0.01$ vs control SHR; ${ }^{\mathrm{d}}-p<0.01$ vs diabetic SHR

Mean \pm SEM are shown except for albuminuria where medians are shown. Data are shown at week 32 of the study period

lar tuft occupied by mesangium was determined. Total mesangial volume was calculated as the product of the mean glomerular volume and the fractional mesangial volume. Each light microscopy section was viewed by an independent observer (B.C.) to determine if there was evidence of glomerulosclerosis.

\section{Statistical analysis}

Statistical analysis of variables was performed by analysis of variance with or without repeated measures using the Clear Lake Research ANOVA Program, Houston, Tex, USA. Albuminuria data were analysed after logarithmic transformation.

\section{Results}

\section{Weight, glycaemic control and blood-pressure (Table 1)}

All diabetic animals gained less weight than their normoglycaemic controls $(p<0.001)$. Enalapril therapy did not influence body weight in any of the groups. There was no significant difference in the degree of hyperglycaemia between the SHR and WKY strains or between untreated and enalapril treated rats. Enalapril therapy was associated with a significant reduction in bloodpressure over the whole study period in diabetic WKY, control SHR and diabetic SHR.

\section{Albuminuria}

In diabetic WKY, albuminuria began to increase after about 24 weeks of diabetes and reached a median of $2.5 \mathrm{mg} / 24 \mathrm{~h}$ at week 32 . This level was much lower than in both diabetic and control SHR. Nevertheless, in diabetic WKY, Enalapril prevented this increase in albuminuria when compared to untreated rats $(p<0.02)$. In diabetic SHR, there was an exponential increase in albuminuria over the study period to $62 \mathrm{mg} / 24 \mathrm{~h}$ at week 32 , which was significantly higher than in control SHR or diabetic WKY. Enalapril therapy in diabetic SHR was associated with the development of less albuminuria than in untreated diabetic SHR $(p<0.001)$. Albuminuria was lower in enalapril treated diabetic SHR than in untreated diabetic SHR from week 8 onwards and remained lower at all further time points. In control SHR, enalapril treatment was also associated with a significant decrease in albuminuria over the whole study period $(p<0.02)$.

\section{Glomerular Ultrastructure}

Enalapril treatment decreased GBM thickness in diabetic WKY, in diabetic SHR and also in control SHR (all groups, $p<0.01$ ) (Fig.1). Enalapril therapy did not influence glomerular volume in diabetic WKY or diabetic SHR when compared to their appropriate group. Enalapril therapy significantly reduced glomerular volume in control SHR $(p<0.01)$. Treatment with enalapril did not alter fractional mesangial volume in either the diabetic strain or in control SHR. The effects of enalapril on total mesangial volume could be explained by the changes in glomerular volume in the SHR strain. There was no evidence of glomerulosclerosis in any of the groups studied.

\section{Discussion}

This appears to be the first study to demonstrate that ACE inhibition prevents GBM thickening in experimental diabetes. It appears that this effect of ACE inhibition is independent of the presence of genetic hypertension since prevention of GBM thickening was documented not only in the hypertensive but also in the normotensive, diabetic animals. Furthermore, the effects of enalapril in preventing GBM thickening were also detected in the non-diabetic animals suggesting that this effect is not confined to diabetes. It is difficult to determine if the effects of enalapril on glomerular ultrastructure and albuminuria are related to specific effects of the ACE inhibitor or to its action as a hypotensive agent, since enalapril decreased blood-pressure significantly in all groups including the normotensive, diabetic animals. The possibility needs to be considered that ACE inhibitors act not only by reducing systemic but also intraglomerular pressure $\left(\mathrm{P}_{\mathrm{GC}}\right)$ since $\mathrm{ACE}$ inhibitors reduce $\mathrm{P}_{\mathrm{GC}}$ by preventing Angiotensin II medi- 

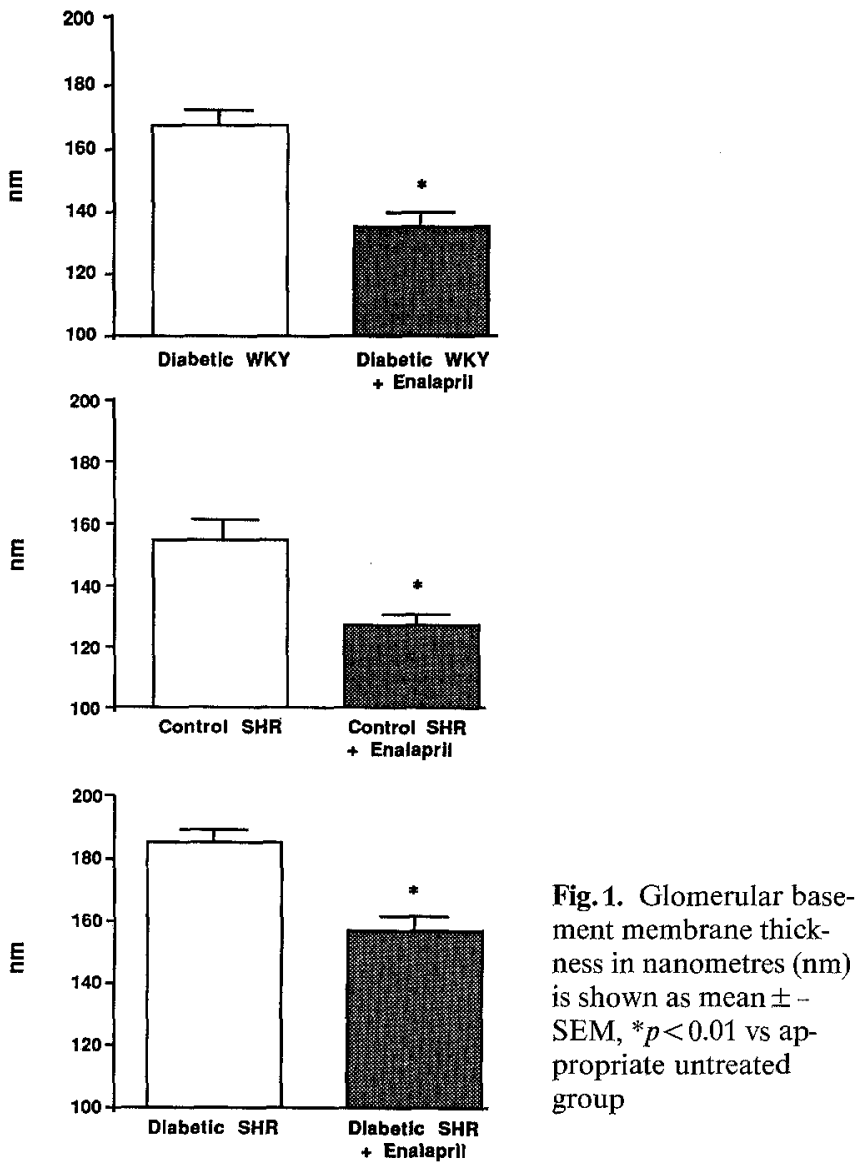

ated efferent arteriolar vasoconstriction [3]. Since micropuncture studies were not performed in the present study, it is difficult to assess the state of intrarenal haemodynamics in the models described. In previous studies, diabetic SHR have had increased $\mathrm{P}_{\mathrm{GC}}$ when compared to diabetic WKY [6] and enalapril therapy in the normotensive Munich-Wistar diabetic rat has been shown to reduce $P_{\mathrm{GC}}$ [3]. However, these changes in $\mathrm{P}_{\mathrm{GC}}$ were detected in insulin-treated diabetic rats and cannot be directly extrapolated to the non-insulin treated diabetic rats in the present study since micropuncture studies in non-insulin treated diabetic rats have in general normal or decreased $\mathrm{P}_{\mathrm{GC}}$ [7].

It has been suggested that capillary hydrostatic pressure is an important determinant of basement membrane thickness and that increases in intravascular pressure result in capillary distension with stimulation of basement membrane synthesis, increased vascular permeability and leakage of plasma constituents [8]. Direct effects of angiotensin II on the glomerular permeability and filtration of proteins or effects of ACE inhibitors on other vasoactive compounds such as kinins or prostaglandins also need to be considered as possible explanations for the effects of enalapril on both GBM thickening and urinary albumin excretion.

ACE inhibition did not significantly influence fractional mesangial volume in diabetic WKY or SHR, or control SHR. ACE inhibitors do not reduce glomerular flow significantly in experimental diabetes [3]. If flow is an important determinant of mesangial expansion this would partly explain the failure of enalapril to influence mesangial expansion. Another possibility is that metabolic rather than haemodynamic factors predominate in diabetes related mesangial expansion. In the present study, enalapril therapy did not influence glycaemic control.

In experimental studies, preliminary reports indicate a possible specific benefit of ACE inhibitors on renal function and structure which may be specific to this class of antihypertensive agents $[9,10]$. However, no direct comparisons have been performed with conventional hypotensive agents in man.

Acknowledgements. This work was supported by grants from the Juvenile Diabetes Foundation International, the Australian Kidney Foundation and the National Health \& Medical Research Council of Australia.

\section{References}

1. Hommel E, Parving HH, Mathiesen E, Edsberg B, Nielsen MD, Giese J (1986) Effect of captopril on kidney function in insulindependent diabetic patients with nephropathy. $\mathrm{Br}$ Med J 293: 467-470

2. Marre M, Leblanc H, Suarez L, Guyenne T, Menard J, Passa P (1987) Converting enzyme inhibition and kidney function in normotensive diabetic patients with persistent microalbuminuria. $\mathrm{Br}$ Med J 294: 1448-1452

3. Zatz R, Dunn BR, Meyer TW, Anderson S, Rennke HG, Brenner BM (1986) Prevention of diabetic glomerulopathy by pharmacological amelioration of glomerular capillary hypertension. J Clin Invest 77: 1925-1930

4. Cooper ME, Allen TJ, O'Brien RC, Macmillan PA, Clarke B, Jerums $G$, Doyle AE (1988) Effects of genetic hypertension on diabetic nephropathy in the rat - functional and structural characteristics. J Hypertens 6: 1009-1016

5. Cooper ME, Allen TJ, Macmillan P, Bach L, Jerums G, Doyle AE (1988) Genetic hypertension accelerates nephropathy in the streptozotocin diabetic rat. Am J Hypertens 1: 5-10

6. Bank N, Klose R, Aynedjian HS, Nguyen D, Sablay LB (1987) Evidence against increased glomerular pressure initiating diabetic nephropathy, Kidney Int 31: 898-905

7. Hostetter TH, Troy JL, Brenner BM (1981) Glomerular hemodynamics in experimental diabetes mellitus. Kidney Int 19: 410-415

8. Williamson JR, Kilo C (1977) Current status of capillary basement-membrane disease in diabetes mellitus. Diabetes 26: 65-73

9. Jackson B, Debrevi L, Whitty M, Johnston CI (1986) Progression of renal disease: effects of different classes of antihypertensive therapy. J Hypertens 4 [Suppl 5]: S269-271

10. Anderson S, Riley SL, Rennke HG, Garcia DL, Brenner BM (1989) Superiority of Captopril over combination triple therapy in arresting diabetic glomerulopathy in the rat. Kidney Int 35: 422 (Abstract)

Received: 25 January 1989

and in revised form: 14 March 1989

Dr. M.E.Cooper

Endocrine Unit

Department of Medicine

Austin Hospital

Heidelberg, Victoria

Australia 